\title{
Zur Kristallstruktur der intermetallischen Phasen MoAs und $\mathrm{Mo}_{5} \mathrm{As}_{4}{ }^{*}$
}

\author{
H. KANDLer und B. ReisS \\ Forschungslaboratorium der Siemens-Schuckertwerke AG, Erlangen \\ (Z. Naturforschg. 21 a, 549-554 [1966]; eingegangen am 1. März 1966)
}

Herrn Prof. Dr. Trendelenburg zum 70. Geburtstag gewidmet

\begin{abstract}
Es wird ein Verfahren zur Herstellung kleiner Einkristalle von MoAs und $\mathrm{Mo}_{5} \mathrm{As}_{4}$ durch gerichtete Erstarrung von GaAs-(Mo, As)-Eutektika beschrieben. Die Kristallstrukturen von MoAs und $\mathrm{Mo}_{5} \mathrm{As}_{4}$ wurden auf röntgenographischem Weg an Hand von Einkristallen und Pulverproben untersucht. MoAs kristallisiert im MnP-Struktur-Typ mit den Gitterkonstanten $a=5,97_{8} \AA, b=3,36_{0} \AA$, $c=6,41_{5} \AA$. $\mathrm{Mo}_{5} \mathrm{As}_{4}$ besitzt' eine $\mathrm{Ti}_{5} \mathrm{Te}_{4}$-Struktur; die Gitterkonstanten betragen: $a=9,59_{1} \AA$, $c=3,28_{1} \AA$.

Die Struktur von $\mathrm{Mo}_{5} \mathrm{As}_{4}$ wird diskutiert.
\end{abstract}

Im Zusammenhang mit Arbeiten über den gerichteten Einbau von Übergangsmetallphasen in $\mathrm{A}^{\mathrm{III}} \mathrm{B}^{\mathrm{V}}$. Verbindungen wurde von Reiss und RenNer ${ }^{1}$ das System GaAs - (Mo, As) untersucht. Dabei gelang es, aus gerichtet erstarrten Eutektika dieses Systems nadelförmige Einkristalle zweier verschiedener Molybdänarsenide zu isolieren, deren Zusammensetzung beim Verhältnis 1:1 lag.

Die eutektische Konzentration des Systems $\mathrm{GaAs}-(\mathrm{Mo}, \mathrm{As})$ liegt nach chemischen Analysen bei 9,5\% MoAs-Gehalt. Zur Herstellung des Eutektikums wurden die für die Bildung von $\mathrm{GaAs}$ und MoAs benötigten Mengen der Ausgangselemente ${ }^{2}$ in Quarzschiffen, die sich in evakuierten, abgeschmolzenen Quarzampullen befanden, zusammengeschmolzen. Es erwies sich als zweckmäßig, vor der Reaktion mit Arsen die beiden Metalle Molybdän und Gallium miteinander zu legieren. GaAs schmilzt bei $1236{ }^{\circ} \mathrm{C}$ unter einem As-Gleichgewichtsdruck von 0,9 atm. Dieser Druck wurde bei unseren Versuchen aufrechterhalten, indem überschüssiges Arsen eingewogen und in eine genau geregelte Temperaturzone der Reaktionsampulle gebracht wurde (sogen. 2-Temperaturen-Verfahren). Die Erstarrungsgeschwindigkeit der Schmelze betrug ca. 0,5 mm/min.

Durch Lösen der GaAs-Matrix in Brom-MethanolGemischen bei $50-60{ }^{\circ} \mathrm{C}$ konnten die ausgeschiedenen Kristalle der (Mo, As)-Phase isoliert werden. Ein geringer Angriff des Lösungsmittels auf das Molybdänarsenid störte bei den weiteren Untersuchungen nicht.

\footnotetext{
* Auszugsweise vorgetragen auf der 8. Diskussionstagung der Sektion für Kristallkunde in der Deutschen Mineralogischen Gesellschaft, Marburg 1965.
}

Unter den oben genannten Bedingungen wurden zwei verschiedene Molybdänarsenide, MoAs und $\mathrm{Mo}_{5} \mathrm{As}_{4}$, erhalten. Durch Erhöhung des Arsendampfdruckes von 0,9 auf $1,1-1,5$ atm gelang es, das Monoarsenid allein herzustellen. Die aus dem Eutektikum isolierten Kristallnadeln hatten Durchmesser von $1-2 \mu \mathrm{m}$ und waren im Mittel $100 \mu \mathrm{m}$ lang. Für die kristallographischen Untersuchungen war es jedoch wünschenswert, Kristalle mit Durchmessern zwischen 100 und $300 \mu \mathrm{m}$ zur Verfügung zu haben. Eine Möglichkeit zur Züchtung solcher Kristalle bietet die Erstarrung von Schmelzen übereutektischer Zusammensetzung. Die in diesem Fall auftretende

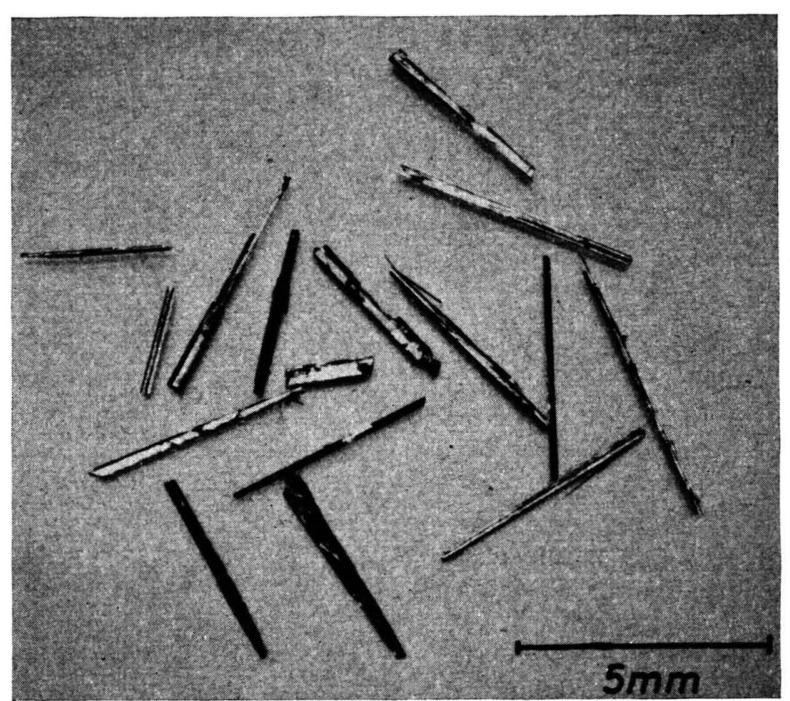

Abb. 1. $\mathrm{Mo}_{5} \mathrm{As}_{4}-$ Kristalle.

1 B. Reiss u. Th. Renner, Z. Naturforschg. 21 a, 546 [1966].

2 Reinheit der Ausgangsmaterialien: Ga 99,999\%, As 99,999\%, Mo (Pulver) 99,99\%. 
Primärausscheidung des Molybdänarsenids am Barrenanfang ließ sich zur Herstellung größerer Kristalle ausnutzen. Aus Schmelzen mit ca. 12\% MoAs konnten Kristalle mit einigen zehntel Millimetern Dicke und mehreren Millimetern Länge erhalten werden (Abb. 1).

\section{Die Kristallstruktur von MoAs}

Für die Strukturbestimmung wurden von Einkristallnadeln Drehkristall- und Weissenberg-Diagramme mit $\mathrm{MoK}_{a}$-Strahlung aufgenommen. Aus den Reflexintensitäten und der Symmetrie des reziproken Gitters ergab sich, daß die Nadeln im orthorhombischen System kristallisieren. Ein Vergleich der Gitterkonstanten mit denen bekannter Arsenide, die ebenfalls im orthorhombischen System kristallisieren, führte zu der Vermutung, daß MoAs im B31-(MnP)-Typ kristallisiert. Um unsere Annahme zu überprüfen, wurden die beobachteten und die berechneten Linienintensitäten von Pulverdiagrammen verglichen. Für die Aufnahme der Diagramme mit dem S i e mens-Zählrohrgoniometer wurde $\mathrm{CuK}_{a^{-}}$ Strahlung verwendet. Der Berechnung der Linienintensitäten wurde das Modell der MnP-Struktur ${ }^{3}$ zugrunde gelegt, wonach MoAs in der Raumgruppe $\mathrm{D}_{2 \mathrm{~h}}^{16}$ - Pnma kristallisiert und die Atome auf folgende Punktlagen verteilt sind:

$$
\begin{aligned}
4 \text { Mo in } 4 \text { (c) }: \quad & x, 1 / 4, z ; \bar{x}, 3 / 4, \bar{z} ; \\
& 1 / 2-x, 3 / 4,1 / 2+z ; \\
& 1 / 2+x, 1 / 4,1 / 2-z ; \\
& \text { mit } x=0,01 \text { und } z=0,19 ;
\end{aligned}
$$

4 As in $4(\mathrm{c})$ : wie oben,

$$
\text { mit } x=0,20 \text { und } z=0,58 \text {. }
$$

An Hand dieser Parameter wurden die Intensitäten nach der Beziehung

$\begin{aligned} & I_{h k l} & =K \cdot P \cdot L \cdot H \cdot\left|F_{h k l}\right|^{2} \\ \text { mit } & F_{h k l} & =\sum_{n=1}^{N} f_{n} \exp \left\{2 \pi i\left(h x_{n}+k y_{n}+l z_{n}\right)\right\}\end{aligned}$

berechnet.

Es bedeutet:

$I_{h k l}:$ Intensität der RönTGEN-Interferenz mit den Millerschen Indizes $h, k, l$,

$K$ : Konstante,

$P$ : Polarisationsfaktor,

$L$ : LoRENTz-Faktor,

$H$ : Häufigkeitsfaktor,

$F$ : Strukturamplitude,

$f_{n}:$ Atomformfaktor,

$x_{n}, y_{n}, z_{n}:$ Koordinaten des $n$-ten Atoms,

$N$ : Zahl der Atome in der Elementarzelle.

\begin{tabular}{|c|c|c|c|c|}
\hline$h k l$ & $d_{\text {beob. }}$ & $d_{\text {berech. }}$ & $I_{\text {beob. }}$ & $I_{\text {berech. }}$ \\
\hline 101 & 4,372 & 4,3728 & 7,0 & 4,7 \\
\hline 011 & 2,978 & 2,9764 & 32,3 & 30,1 \\
\hline 102 & 2,826 & 2,8265 & 24,2 & 36,6 \\
\hline 111 & 2,663 & 2,6645 & 44,2 & 53,7 \\
\hline 210 & 2,235 & 2,2334 & 23,5 & 13,4 \\
\hline 202 & 2,186 & 2,1869 & 57,1 & 42,1 \\
\hline 112 & 2,163 & 2,1629 & 59,6 & 56,8 \\
\hline 211 & 2,110 & 2,1093 & 100,0 & 100,0 \\
\hline 103 & 2,013 & 2,0134 & 23,5 & 24,2 \\
\hline 301 & 1,905 & 1,9033 & 25,7 & 19,3 \\
\hline 013 & 1,803 & 1,8039 & 18,4 & 27,6 \\
\hline 203 & 1,739 & 1,7392 & 6,5 & $4,3\}$ \\
\hline 113 & - & 1,7371 & - & $0,7\}$ \\
\hline 020 & 1,681 & 1,6800 & 19,6 & 23,5 \\
\hline 311 & 1,658 & 1,6560 & 5,4 & 6,1 \\
\hline 104 & 1,548 & 1,5489 & 3,9 & $3,9\}$ \\
\hline 213 & - & 1,5445 & - & $0,8\}$ \\
\hline 400 & 1,494 & 1,4947 & 4,8 & 7,1 \\
\hline 220 & 1,464 & 1,4646 & 5,0 & 1,3 \\
\hline 014 & 1,445 & 1,4473 & - & 0 \\
\hline 122 & - & 1,4442 & 6,8 & $7,3\}$ \\
\hline 114 & 1,407 & 1,4067 & 6,1 & $9,3\}$ \\
\hline 313 & - & 1,3375 & - & $7,8)$ \\
\hline 411 & 1,335 & 1,3358 & 14,9 & $13,7\}$ \\
\hline 222 & - & 1,3323 & - & $0,3]$ \\
\hline 214 & - & 1,3027 & $=$ & 0,3 \\
\hline 123 & 1,290 & 1,2899 & 5,6 & $9,2\}$ \\
\hline 320 & - & 1,2845 & - & $0\}$ \\
\hline 321 & 1,259 & 1,2595 & 11,6 & $8,1)$ \\
\hline 412 & - & 1,2565 & - & $3,2\}$ \\
\hline 105 & - & 1,2544 & - & $6,4]$ \\
\hline 205 & - & 1,1790 & - & $0,3)$ \\
\hline 501 & - & 1,1756 & - & 0,3 \\
\hline 115 & - & 1,1752 & - & 3,2 \\
\hline 314 & 1,171 & 1,1711 & 15,6 & $17,9)$ \\
\hline
\end{tabular}

In Tab. 1 sind die beobachteten und berechneten Netzebenenabstände ( $d$-Werte) und die beobachteten und berechneten relativen Linienintensitäten $(I)$ zusammengestellt. Die Übereinstimmung zwischen

Tab. 1. Pulverdiagramm von $\mathrm{MoAs}\left(\mathrm{CuK}_{\alpha}\right.$-Strahlung).

Raumgruppe: $\mathrm{D}_{2 \mathrm{~h}}^{16}-$ Pnma, Nr. 62 der Internationalen Tabellen ${ }^{4}$

Gitterkonstanten:

$$
a=5,97_{8} \AA, \quad b=3,36_{0} \AA, \quad c=6,41_{5} \AA .
$$

Dichte (röntgenographisch) : $8,81 \mathrm{~g} \mathrm{~cm}^{-3}$.

Punktlagen :

4. Mo in 4 (c) : $\quad x, 1 / 4, \quad z ; \quad \bar{x}, 3 / 4, \bar{z}$;

$$
\begin{aligned}
& 1 / 2-x, 3 / 4, \quad 1 / 2+z ; \\
& 1 / 2+x, \quad 1 / 4, \quad 1 / 2-z . \\
& \text { mit } x=0,01 \text { und } z=0,19 ;
\end{aligned}
$$

4 As in 4 (c) : wie oben, mit $x=0,20$ und $z=0,58$. Strukturtyp: B 31-(MnP)-Typ.

Tab. 2. Ergebnisse der Strukturbestimmung von MoAs.

3 W. B. Pearson, A Handbook of Lattice Spacings and Structures of Metals and Alloys, Pergamon Press, London 1958.

4 International Tables for X-ray Crystallography, The Kynoch Press, Birmingham 1952. 
berechneten und beobachteten Werten ist im Rahmen der Meßgenauigkeit recht gut.

In Tab. 2 sind die Ergebnisse der röntgenographischen Strukturbestimmung zusammengefaßt.

\section{Die Kristallstruktur von $\mathbf{M o}_{5} \mathbf{A s}_{4}$}

Von dieser Mo - As-Phase war nach vorläufigen Untersuchungen mit der RöNTGEN-Mikrosonde nur bekannt, daß die Zusammensetzung etwas vom Verhältnis $1: 1 \mathrm{zu}$ höheren Molybdänkonzentrationen hin verschoben ist.

Für die Strukturanalyse wurden von gut ausgebildeten, nadelförmigen Einkristallen mit $\mathrm{MoK}_{a}$-Strahlung Drehkristall- und Weissenberg-Diagramme längs und senkrecht zur Nadelachse aufgenommen. Die Aufnahmen zeigten die Laue-Symmetrie $4 / \mathrm{m}$. Es wurden nur Reflexe beobachtet, für die die Beziehung

$$
h+k+l=2 n
$$

gilt. Die höchstsymmetrische Raumgruppe, die damit übereinstimmt, ist $\mathrm{C}_{4 \mathrm{~h}}^{5}-\mathrm{I} 4 / \mathrm{m}$. Für die Gitterkonstanten wurden aus Drehkristall- und Pulveraufnahmen die Werte $a=9,59_{1} \AA, c=3,28_{1} \AA$ bestimmt.

Die Intensitäten der $h k 0$ - und $h k l$-Reflexe wurden an Hand der Weissenberg-Aufnahmen visuell geschätzt und daraus der Betrag der Strukturamplituden berechnet. Eine Projektion der PattersonFunktion ${ }^{5}$

$$
P(u, v)=K \sum_{h} \sum_{k}\left|F_{h k 0}\right|^{2} \cos 2 \pi(h u+k v)
$$

auf die 001-Ebene wurde berechnet, wobei $u, v$ die laufenden Koordinaten und $K$ eine Konstante sind. Die Interpretation dieser Funktion, die eine Abbildung aller interatomarer Vektoren darstellt, ergab ein Strukturmodell, bei dem sich $8 \mathrm{Mo}$ - und $8 \mathrm{As}$ Atome auf je einer 8-zähligen Punktlage befinden. Für einen kleineren Mo-Überschuß kommt dann nur mehr eine 2-zählige Punktlage in Frage. Damit erhält man die Summenformel $\mathrm{Mo}_{5} \mathrm{As}_{4}$.

Das aus der Patterson-Funktion abgeleitete Strukturmodell ist mit der Struktur des $\mathrm{Ti}_{5} \mathrm{Te}_{4}$, die von GRønvold et al. ${ }^{6}$ aufgeklärt wurde, isotyp.

5 H. Lipson u. W. Cochran, The Determination of Crystal Structures, Bell \& Sons, London 1953.

6 F. Grønvold, A. Kjekshus u. F. RaAum, Acta Cryst. 14, 930 [1961].
Ein Vergleich der beobachteten Strukturamplituden mit den an Hand der Parameter der $\mathrm{Ti}_{5} \mathrm{Te}_{4}$ Struktur berechneten ergab nur eine schlechte Utbereinstimmung. Die Parameter der $\mathrm{Ti}_{5} \mathrm{Te}_{4}$-Struktur wurden der Rechnung zugrunde gelegt, weil angenommen wurde, da $\beta$ sie genauer seien als die aus der Patterson-Funktion entnommenen.

Der $R_{1}$-Faktor,

$$
R_{1}=\frac{\Sigma\left\|F_{0}|-| F_{\mathrm{c}}\right\|}{\Sigma\left|F_{0}\right|},
$$

der als Maß für die Übereinstimmung zwischen beobachteten $\left(F_{0}\right)$ und berechneten $\left(F_{\mathrm{c}}\right)$ Strukturamplituden gilt und für vollständige Übereinstimmung Null ist, wurde für $30 h k 0$-Reflexe zu 0,51 berechnet.

Es war anzunehmen, daß die beobachteten Unterschiede zwischen den berechneten und beobachteten Strukturamplituden nur auf kleine Abweichungen zwischen den Atomlagen der Mo- bzw. As-Atome, und denen der entsprechenden $\mathrm{Ti}$ - und Te-Atome, zurückzuführen sind.

Zur Verbesserung der Übereinstimmung von Modell und Struktur wurde ein ALGOL-Programm geschrieben, das es ermöglicht, mit Hilfe einer elektronischen Rechenmaschine durch schrittweise Variation freiwählbarer Parameter den $R_{1}$-Faktor zu verkleinern.

Die Rechnung wurde in mehreren Schritten durchgeführt: Im ersten wurden nur $30 h k 0$-Reflexe berücksichtigt und die $x, y$-Parameter variiert, im zweiten alle beobachteten $h k 0$-Reflexe in die Rechnung einbezogen und ein allen Atomen gemeinsamer, isotroper Temperaturfaktor ${ }^{7}$ eingeführt. Im dritten Schritt wurde der $R_{1}$-Faktor für die $h k l$-Reflexe berechnet, bei denen neben den $x, y$-Koordinaten auch die $z$-Koordinaten in die Rechnung eingehen.

Zum Schluß wurden unter Berücksichtigung aller beobachteter Reflexe die $x, y$-Parameter und für jede Atomsorte ein eigener Temperaturfaktor berechnet. Der $R_{1}$-Faktor beträgt 0,16. In Tab. 3 sind die Ergebnisse der Parameter-Verfeinerung zusammengestellt.

Eine Fehlerabschätzung für die verschiedenen Parameter ist wegen der ziemlich großen Unsicher-

7 Nach Debye wird der Einfluß isotroper, thermischer Schwingungen auf den Atom-Formfaktor durch den Ausdruck $f_{T}=f_{T}=0 \exp \left\{-B\left(\sin ^{2} \vartheta\right) / \lambda^{2}\right\}$ berücksichtigt, wobei $B$ der Debyesche Temperaturfaktor und $T$ die absolute Temperatur ist. 


\begin{tabular}{|c|c|c|c|c|c|c|c|}
\hline \multirow{2}{*}{ Indizes } & $\begin{array}{c}\text { Zahl der } \\
\text { Reflexe }\end{array}$ & $x_{\text {Mo }}$ & $y_{\text {Mo }}$ & $x_{\text {As }}$ & $y_{\text {As }}$ & $B$ & $R_{1} \cdot 10^{2}$ \\
\cline { 1 - 6 }$h k 0$ & 30 & 0,298 & 0,378 & 0,056 & 0,288 & $-17,5$ \\
$h k 0$ & 61 & 0,301 & 0,380 & 0,058 & 0,289 & 0.0 & 16,5 \\
$h k 1$ & 57 & 0,301 & 0,380 & 0,058 & 0,289 & 0,0 & $0,15(\mathrm{Mo})$ \\
$h k 0$ & 118 & 0,3006 & 0,3792 & 0,0572 & 0,2886 & $0,0(\mathrm{As})$ & 15,8 \\
$h k 1$ & & & & & & & \\
\hline
\end{tabular}

Tab. 3. Ergebnisse der Parameterverfeinerung.

\begin{tabular}{|c|c|c|c|c|c|c|c|c|c|c|c|c|c|c|c|}
\hline$h k l$ & $\left|F_{0}\right|$ & $F_{\mathrm{c}}$ & & $k$ & & $\left|F_{0}\right|$ & $F_{\mathrm{c}}$ & & $k \quad l$ & $\left|F_{0}\right|$ & $F_{\mathrm{c}}$ & & $k l$ & $\left|F_{0}\right|$ & $F_{\mathrm{c}}$ \\
\hline 200 & 69 & $-54,6$ & 1 & 5 & & 110 & 182,4 & 3 & 110 & 111 & 108,9 & 11 & 41 & 134 & 195,6 \\
\hline 400 & 55 & 44,2 & 3 & 5 & 0 & 108 & 125,5 & 5 & 110 & 105 & $-86,8$ & 15 & 4 & 115 & 110,0 \\
\hline $\begin{array}{lll}6 & 0 & 0\end{array}$ & 20 & 17,0 & 5 & 5 & 0 & 94 & $-77,7$ & 7 & 110 & 84 & $-101,1$ & 6 & 51 & 55 & $-204,7$ \\
\hline 800 & 30 & $-35,3$ & 7 & 5 & 0 & 178 & 218,4 & 9 & 110 & 129 & 150,3 & 8 & 51 & 76 & 49,2 \\
\hline 1000 & 108 & 132,5 & 9 & 5 & 0 & 88 & 78,7 & 2 & 120 & 118 & 107,9 & 10 & 51 & 92 & 86,8 \\
\hline 1200 & 140 & $-159,8$ & 11 & 5 & 0 & 126 & 111,5 & 12 & 120 & 129 & 140,2 & 12 & 51 & 86 & 81,9 \\
\hline 1400 & 125 & 87,2 & 2 & 6 & 0 & 286 & 289,4 & 1 & 130 & 33 & $-54,0$ & 14 & 51 & 108 & 98,0 \\
\hline 1600 & 117 & 104,4 & 4 & 6 & 0 & 53 & 32,2 & 5 & 130 & 35 & 68,4 & 7 & 61 & 71 & 45,4 \\
\hline 110 & 87 & 96,6 & 6 & 6 & 0 & 72 & 44,8 & 7 & 130 & 106 & 105,3 & 9 & 61 & 68 & 52,8 \\
\hline $\begin{array}{lll}3 & 1 & 0\end{array}$ & 51 & 49,8 & 8 & 6 & 0 & 108 & 100,1 & 2 & 140 & 35 & 59,4 & 2 & 71 & 91 & 72,1 \\
\hline $\begin{array}{lll}5 & 1 & 0\end{array}$ & 95 & $-101,5$ & 10 & 6 & 0 & 30 & 20 & 4 & 140 & 118 & $-85,8$ & 6 & 71 & 6 & $-72,6$ \\
\hline 710 & 71 & $-65,2$ & 12 & 6 & 0 & 94 & 70 & 6 & 140 & 126 & 132,5 & 8 & 71 & 146 & 165,0 \\
\hline $\begin{array}{lll}9 & 1 & 0\end{array}$ & 116 & 140,9 & 14 & 6 & 0 & 146 & $-146,8$ & 1 & 150 & 76 & 48,5 & 10 & 71 & 133 & $-124,1$ \\
\hline 1110 & 139 & 152,9 & 1 & 7 & 0 & 249 & 261,4 & 5 & $\begin{array}{ll}0 & 1\end{array}$ & 70 & $-67,2$ & 1 & 81 & 88 & 76,1 \\
\hline 220 & 195 & $-89,7$ & 3 & 7 & 0 & 27 & 59,7 & 7 & $\begin{array}{ll}0 & 1\end{array}$ & 88 & 85,0 & 5 & 81 & 119 & $-109,0$ \\
\hline 420 & 20 & 41,9 & 5 & 7 & 0 & 138 & 161,0 & 9 & $\begin{array}{lll}0 & 1\end{array}$ & 140 & 171,4 & 9 & 81 & 144 & 154,3 \\
\hline 620 & 85 & $-83,5$ & 7 & 7 & 0 & 132 & $-133,5$ & 13 & 01 & 128 & 141,3 & 2 & 91 & 154 & 245,6 \\
\hline 820 & 112 & 104,1 & 9 & 7 & 0 & 74 & $-73,1$ & 17 & 01 & 86 & 97,6 & 6 & 91 & 92 & 51,1 \\
\hline 1020 & 125 & 119,9 & 11 & 7 & 0 & 119 & 131,5 & 4 & 11 & 35 & $-35,6$ & 8 & 91 & 86 & 94,1 \\
\hline 1220 & - & 18,0 & 2 & 8 & 0 & 88 & $-82,3$ & 6 & 11 & 74 & 106,5 & 12 & 91 & 157 & 152,5 \\
\hline 1420 & 86 & 82,7 & 4 & 8 & 0 & 25 & 29,8 & 8 & 11 & 39 & 52,6 & 14 & 91 & 151 & $-134,3$ \\
\hline 1620 & 151 & $-167,7$ & 6 & 8 & 0 & 88 & 92, & 12 & 11 & 103 & 109,0 & 1 & 101 & 85 & 93,4 \\
\hline 130 & 14 & $-21,4$ & 8 & 8 & 0 & 65 & 41,3 & 14 & 11 & 138 & 127,8 & 3 & 101 & 102 & 101,7 \\
\hline $\begin{array}{lll}3 & 3 & 0\end{array}$ & 216 & 260,5 & 10 & 8 & 0 & 124 & 124,4 & 5 & 21 & 44 & 31,2 & 5 & 101 & 149 & 136,2 \\
\hline 530 & 111 & 135,1 & 1 & 9 & 0 & 26 & $-44,2$ & 11 & 21 & 112 & 104,3 & 9 & 101 & 79 & $-74,1$ \\
\hline 730 & 99 & 91,6 & 3 & 9 & 0 & 92 & $-98,4$ & 13 & 21 & 83 & $-84,7$ & 4 & 111 & 101 & $-95,3$ \\
\hline $\begin{array}{lll}9 & 3 & 0\end{array}$ & 110 & $-101,1$ & 5 & 9 & 0 & 205 & 236,3 & 15 & 21 & 67 & 38,3 & 6 & 111 & 164 & 151,5 \\
\hline 240 & 20 & $-29,9$ & 7 & 9 & 0 & 30 & 33,0 & 4 & 31 & 96 & 69,7 & 8 & 111 & 89 & $-86,6$ \\
\hline 440 & 20 & 31,2 & 9 & 9 & 0 & 141 & 149,8 & 10 & 31 & 74 & 75,4 & 3 & 121 & 109 & $-107,2$ \\
\hline 640 & 77 & $-57,8$ & 2 & 10 & 0 & 115 & 102,2 & 12 & $\begin{array}{ll}31 \\
1\end{array}$ & 87 & $-71,1$ & 5 & 121 & 61 & 78,2 \\
\hline 840 & 118 & 171,6 & 4 & 10 & 0 & 29 & 31,1 & 16 & 31 & 78 & 72,1 & 9 & 121 & 117 & 132,2 \\
\hline 1040 & 136 & $-142,3$ & 6 & 10 & 0 & 37 & 53 & 3 & 41 & 39 & $-34,8$ & 10 & 131 & 123 & 125,6 \\
\hline 1240 & 112 & 75,2 & 8 & 10 & 0 & 121 & 85,9 & 5 & 41 & 77 & 61,3 & 1 & 161 & 126 & $-111,6$ \\
\hline 1440 & 133 & 130,8 & & & & & & 7 & 41 & 138 & $-154,1$ & & & & \\
\hline
\end{tabular}

Tab. 4. Vergleich der beobachteten und berechneten Strukturamplituden von $\mathrm{Mo}_{5} \mathrm{As}_{4}$.

heiten bei der visuellen Bestimmung der Reflexintensitäten und wegen des Verzichtes auf Absorptions- und Extinktionskorrekturen nur schwer möglich. Trotzdem kann man mit einiger Sicherheit sagen, daß die mittleren Fehler in den Atomkoordinaten sicher kleiner als $0,05 \AA$ sind. Die Temperaturfaktoren $(B)$ für Molybdän und Arsen haben sehr wahrscheinlich keine physikalische Bedeutung.

In der Tab. 4 sind die beobachteten und berechneten Strukturamplituden von $\mathrm{Mo}_{5} \mathrm{As}_{4}$ zusammengestellt. Die Ergebnisse der Strukturbestimmung sind in Tab. 5 zusammengefaßt.
Raumgruppe: $\mathrm{C}_{4 \mathrm{~h}}^{5}-\mathrm{I} 4 / \mathrm{m}$, Nr. 87 der Internationalen Tabellen ${ }^{4}$

Gitterkonstanten:

$$
a=9,59_{1} \AA, \quad c=3,28_{1} \AA, \quad c / a=0,342 .
$$

Dichte (röntgenographisch) : $8,58 \mathrm{~g} \mathrm{~cm}^{-3}$.

Punktlagen: $(0,0,0 ; 1 / 2,1 / 2,1 / 2)+$

2 Mo in 2 (a) $0,0,0$;

8 Mo in 8 (h): $x, y, 0 ; \bar{y}, x, 0 ; \bar{x}, \bar{y}, 0 ; y, \bar{x}, 0$ mit $x=0,300_{6}$ und $y=0,379_{2}$;

$8 \mathrm{As}$ in $8(\mathrm{~h}): \quad$ wie oben, mit $x=0,057_{2}$ und $y=0,288_{6}$. Temperaturfaktoren: $B_{\mathrm{Mo}}=0,15 \AA^{2}, \quad B_{\mathrm{As}}=0,0 \AA^{2}$. Struktur-Typ: $\mathrm{Ti}_{5} \mathrm{Te}_{4}$.

Tab. 5. Ergebnisse der Strukturbestimmung von $\mathrm{Mo}_{5} \mathrm{As}_{4}$. 


\section{Diskussion}

Die Struktur von MoAs ist mit der bekannten MnP-Struktur (B 31-Typ) isotyp. Damit läßt sich diese Verbindung zwanglos in die Reihe der Monoarsenide der Übergangsmetalle einordnen, die von der VI a- bis VII a-Gruppe des Periodensystems im B 31- oder B 8-Typ (NiAs-Struktur) kristallisieren ${ }^{8}$.

Die Struktur von $\mathrm{Mo}_{5} \mathrm{As}_{4}$, die an Hand von Einkristalluntersuchungen bestimmt und deren Parameter verfeinert wurden, baut sich aus durchgehenden Säulen von innenzentrierten Molybdänquadern auf, die untereinander durch Arsenbrücken verknüpft sind. Abb. 2 zeigt eine Projektion der $\mathrm{Mo}_{5} \mathrm{As}_{4}$ Struktur auf die 001-Ebene.

Ein Vergleich der Atomabstände im $\mathrm{Mo}_{5} \mathrm{As}_{4}$ (Tab. 6) mit denen im $\mathrm{Ti}_{5} \mathrm{Te}_{4}$ zeigt, daß die $\mathrm{Mo}-$ As-Bindung, wie die entsprechende $\mathrm{Ti}-\mathrm{Te}$-Bindung um rund $12 \%$ gegenüber der Summe der Metallradien für $12 \mathrm{er}$-Koordination nach $\mathrm{P}_{\mathrm{AULING}}{ }^{9}$ verkürzt ist. Die kürzesten Mo-Mo-Abstände, die mit 2,77 $\AA$ genau der Summe der Metallradien für 12erKoordination entsprechen, sind nur wenig (rund 1,8\%) gegenüber den Atomabständen im Molybdänmetall vergrößert. Im $\mathrm{Ti}_{5} \mathrm{Te}_{4}$ weichen dagegen die $\mathrm{Ti}-\mathrm{Ti}$-Abstände etwas stärker (rund $3,9 \%$ ) von den Atomabständen im $\beta$-Titan ab.

\begin{tabular}{|c|c|c|c|c|c|}
\hline $\mathrm{Mo}^{\mathrm{a}}$ & $-8 \mathrm{Mo}$ & $2,77 \AA$ & As & $-2 \mathrm{Mo}$ & $2,52 \AA$ \\
\hline & $-2 \mathrm{Mo}$ & $3,28 \AA$ & & $-2 \mathrm{Mo}$ & $2,67 \AA$ \\
\hline & $-4 \mathrm{As}$ & $2,82 \AA$ & & -1 Mo & $2,49 \AA$ \\
\hline $\mathrm{Mo}^{\mathrm{b}}$ & $-2 \mathrm{Mo}$ & $2,77 \AA$ & & -1 Mo & $2,81 \AA$ \\
\hline & -2 Мо & $3,16 \AA$ & & $-4 \mathrm{As}$ & $3,39 \AA$ \\
\hline & $-2 \mathrm{Mo}$ & $3,13 \AA$ & & $-2 \mathrm{As}$ & $3,28 \AA$ \\
\hline & -2 Mo & $3,28 \AA$ & \multirow{4}{*}{\multicolumn{3}{|c|}{$\begin{array}{l}\text { a Mo auf der } 2 \text { (a) Punkt- } \\
\text { lage } \\
\text { b Mo auf der } 8 \text { (h) Punkt- } \\
\text { lage }\end{array}$}} \\
\hline & $-2 \mathrm{As}$ & $2,52 \AA$ & & & \\
\hline & $-2 \mathrm{As}$ & $2,67 \AA$ & & & \\
\hline & $-1 \mathrm{As}$ & $2,49 \AA$ & & & \\
\hline
\end{tabular}

Tab. 6. Atomabstände in $\mathrm{Mo}_{5} \mathrm{As}_{4}$.

Es ist anzunehmen, daß die Mo-Mo-Abstände und die nahezu kubische Koordination der zentralen Mo-Atome auf einen metallischen Charakter dieser Bindung hinweisen. Die in Richtung der $c$-Achse durchlaufenden Mo-Ketten müßten zu einer deutli-

8 Zum Beispiel H. Boller u. E. Parthe, Acta Cryst. 16, 1095 [1963].

9 L. Pauling, The Nature of the Chemical Bond, Cornell University Press, $3^{\text {rd }}$ edn., Ithaka 1963.

10 K. Selte u. A. Kuekshus, Acta Chem. Scand. 17, 2560 [1963].

11 E. Røst u. L. Guertsen, Z. Anorg. Allg. Chem. 328, 299 [1964].

12 F. Grønvold, H. Haraldson, B. Pedersen u. T. Turte, demnächst, zitiert nach E. Røst et al. ${ }^{11}$. chen Anisotropie der elektrischen Leitfähigkeit führen, da senkrecht zu dieser Richtung keine unmittelbaren Mo-Mo-Verknüpfungen bestehen, sondern die Mo-As-Bindungen überwiegen. Versuche zur Messung der Anisotropie der Leitfähigkeit sind im Gange.

Eine Übersicht über die bekannten $\mathrm{A}_{5} \mathrm{~B}_{4}$-Verbindungen mit $\mathrm{Ti}_{5} \mathrm{Te}_{4}$-Struktur (Tab. 7) zeigt, daß bisher nur Verbindungen beobachtet wurden, bei denen die A-Atome Übergangsmetalle und die B-Atome Elemente der V b- und VIb-Gruppe des Periodensystems sind.

\begin{tabular}{|l|c|c|c|}
\hline \multicolumn{1}{|c|}{$\mathrm{A}_{5} \mathrm{~B}_{4}$} & $a$ & $c / a$ & Literatur \\
\hline $\mathrm{Ti}_{5} \mathrm{Te}_{4}$ & 10,164 & 0,3711 & 6 \\
$\mathrm{Nb}_{5} \mathrm{Te}_{4}$ & 10,321 & 0,3635 & 10 \\
$\mathrm{Nb}_{5} \mathrm{Se}_{4}$ & 9,871 & 0,3498 & 10 \\
$\mathrm{~V}_{5} \mathrm{Se}_{4}$ & 9,294 & 0,3677 & 11 \\
$\mathrm{~V}_{5} \mathrm{~S}_{4}$ & - & - & 12 \\
$\mathrm{Mo}_{5} \mathrm{As}_{4}$ & $9,59_{1}$ & 0,342 & 13,14 \\
$\left(\mathrm{~W}_{4} \mathrm{Ti}_{5} \mathrm{As}_{4}\right.$ & 9,57 & 0,345 & 14 \\
$\mathrm{~V}_{5} \mathrm{Sb}_{4}$ & 9,81 & 0,346 & 15 \\
$\mathrm{Nb}_{5} \mathrm{Sb}_{4}$ & $10,31_{5}$ & 0,346 & 16 \\
$\mathrm{Ta}_{5} \mathrm{Sb}_{4}$ & $10,23_{4}$ & 0,346 & 14 \\
& & & \\
\hline
\end{tabular}

Tab. 7. Verbindungen mit $\mathrm{Ti}_{5} \mathrm{Te}_{4}$-Struktur.

Auf den Zusammenhang zwischen der $\mathrm{Ti}_{5} \mathrm{Te}_{4}$. Struktur mit dem NiAs-Typ wurde bereits von GrøNvolD et al. ${ }^{6}$ hingewiesen, auf den mit dem $\mathrm{Cu}_{2} \mathrm{Sb}$ Typ von Schubert ${ }^{17}$ und Boller et al. ${ }^{14}$.

Weitere, interessante Beziehungen bestehen zu $\mathrm{V}_{4} \mathrm{Zn}_{5}$-Struktur, die von Rossteutscher und SchuBERT ${ }^{18}$ aufgeklärt wurde. In Abb. 2 sind die Projektionen der $\mathrm{Ti}_{5} \mathrm{Te}_{4}$-Struktur und der $\mathrm{V}_{4} \mathrm{Zn}_{5}$-Struktur nebeneinandergestellt. Man sieht, daß man bereits durch eine kleine Drehung der Übergangsmetallquader der $\mathrm{Ti}_{5} \mathrm{Te}_{4}$-Struktur zur $\mathrm{V}_{4} \mathrm{Zn}_{5}$-Struktur kommt, in der $\mathrm{Zn}$ die Rolle der Übergangsmetalle und $\mathrm{V}$ die der B-Atome übernommen hat.

Nach Abschluß der vorliegenden Untersuchungen wurde uns eine Arbeit von Boller und Nowotny ${ }^{14}$ bekannt, in der die Verbindungen MoAs und $\mathrm{Mo}_{5} \mathrm{As}_{4}$ an Hand von Pulverdiagrammen beschrieben wer-

13 Diese Arbeit.

14 H. Boller u. H. Nowotny, Monatsh. Chem. 95, 1272 [1964].

15 H. G. Meissner u. K. Schubert, Z. Metallk. 56, 523 [1965].

16 S. Furuseth u. A. Kuekshus, Acta Chem. Scand. 18, 1180 [1964].

17 K.Schubert, Kristallstrukturen zweikomponentiger Phasen, Verlag Springer, Berlin 1964.

18 W.Rossteutscher u. K.Schubert, Z. Metallk. 55, 617[1964]. 


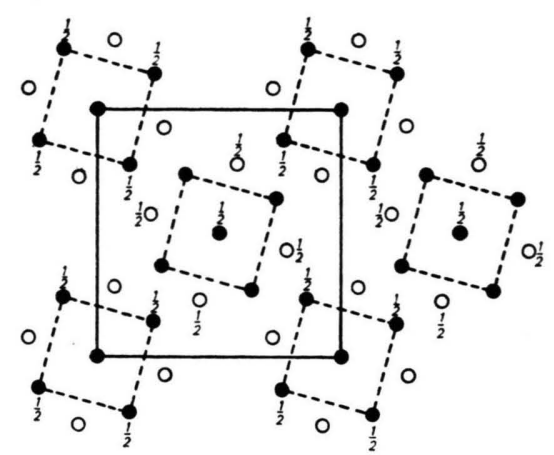

- Mo

$\circ$ As

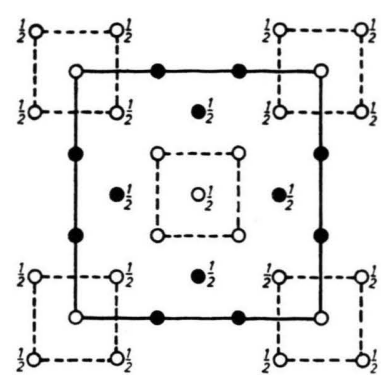

- $v$

$\mathrm{Mo}_{5} \mathrm{As}_{4}$

$\mathrm{V}_{4} \mathrm{Zn}_{5}$

Abb. 2. Projektion der Strukturen von $\mathrm{Mo}_{5} \mathrm{As}_{4}$ und $\mathrm{V}_{4} \mathrm{Zn}_{5}$ auf die 001-Ebene.

den. Die Ergebnisse für MoAs stimmen im wesentlichen mit unseren überein. Allerdings werden die Abweichungen zwischen beobachteten und berechneten Intensitäten kleiner, wenn man der Rechnung, wie von uns angenommen, die Parameter der MnPStruktur zugrunde legt. Für vierzehn gut meßbare Intensitäten errechnet man an Hand unseres nicht verfeinerten Modells einen $R_{1}$-Faktor von 0,12 , mit dem von Boller und Nowotny einen Wert von 0,26.
Für $\mathrm{Mo}_{5} \mathrm{As}_{4}$ wurde von diesen Autoren eine $\mathrm{Ti}_{5} \mathrm{Te}_{4}$ artige Struktur vorgeschlagen, doch war es ihnen an Hand der Pulverdaten nicht möglich zu entscheiden, wie die Molybdän- und Arsenatome auf die beiden $8(\mathrm{~h})$-Punktlagen verteilt sind.

Alle Rechnungen wurden auf der Si emens-Daten. verarbeitungsanlage 2002 ausgeführt, für die ein Satz von ALGOL-Programmen zur röntgenographischen Strukturanalyse geschrieben wurde. 